\title{
An unusual initial presentation of a lung carcinoma with multiple extensive bone metastasis - a diagnostic dilemma
}

\author{
Abeyaratne D D K ${ }^{1}$, Liyanapathirana $C^{1}$, Sivagamaroobasundarie $\mathbf{S}^{1}$, Fonseka $C \mathrm{~L}^{3}$, Shyamali $\mathbf{N ~ L ~}^{1,2}$ \\ Journal of the Ceylon College of Physicians, 2016, 47, 102-105
}

DOI: http://doi.org/10.4038/jccp.v47i2.7793

\begin{abstract}
Background: Bone is a common metastatic site of primary lung carcinoma. But bone metastasis being the initial presentation of primary lung malignancy is rare and only a few cases are reported in literature. We report a case of a primary lung cancer with extensive bone metastasis to skull and long bones, with no clinically or radiologically evident primary tumor.
\end{abstract}

Case presentation: A 45 years old male presented with left lower limb pain for two weeks duration. He also had progressively enlarging two bony lumps on the scalp for six months. He had no other symptoms on inquiry. Examination revealed two large protruding bony lumps on scalp, on posterior vertex and on the left forehead. Respiratory system examination was normal. Skeletal survey showed lytic lesions in right tibia and left humerus with two large bone lesions in the skull extending in to brain matter evident on Contrast CT brain. Chest radiograph revealed no significant lesions. First biopsy of posterior scalp lesion was inconclusive. On further imaging, the contrast enhanced CT chest revealed a small left lung opacity suggestive of bronchogenic carcinoma. Deep FNAC of the posterior scalp lump revealed squamous cell origin, possible metastasis from primary lung carcinoma.

\footnotetext{
${ }^{1}$ University Medical Unit, Colombo South Teaching Hospital, Kalubowila, Sri Lanka.

${ }^{2}$ Department of Medicine, University of Sri Jayewardenepura, Nugegoda, Sri Lanka.

${ }^{3}$ Department of Medicine, Faculty of Medicine, University of Ruhuna, Sri Lanka.
}

E-mail: ajaliyanage@yahoo.com
Conclusion: Our case highlights, that a primary lung carcinoma without clinical or basic radiological evidence can present with large multiple extensive bony metastasis, where early advanced imaging is important to prevent diagnostic dilemmas and therapeutic delay.

Key words: lung carcinoma, bone metastasis, bony lump.

\section{Background}

Bone is the third most frequent site for metastatic deposits after lung and liver. ${ }^{1}$ About $50 \%$ of cancers metastasize to bone. The sites most involved in order of frequency are: spine, pelvis, ribs, skull and proximal long bones. ${ }^{2}$ Bone is a common site of metastasis from lung carcinoma too. The incidence of bone metastases in post-mortem examination in lung cancer was found to be $30 \% .^{3}$

However, metastatic bone disease as the initial presentation of primary lung carcinoma is a rare ${ }^{4,5}$ and only very few cases are reported in literature. But in most of previous case reports, the primary lesion had been evident on basic chest imaging unlike in our patient.

\section{Case report}

A 45 year old male presented with severe pain in the right leg for two months with difficulty in walking. $\mathrm{He}$ also complained of two progressively enlarging lumps on head for four months; one on left forehead and the other on posterior vertex. He had continuous generalized dull headache along with loss of appetite and loss of weight. He did not have fever. In the systemic inquiry, he did not have any respiratory, genitourinary or gastrointestinal symptoms. He had been a heavy smoker in the past for fifteen years but had stopped completely few years back.

On examination, he was pale, cachectic and had severe tenderness over the right proximal tibia. The two 
large lumps on head were irregular and hard. One lump was protruding from left forehead compressing on the left eyelid causing a partial ptosis on that side, and the other lump was at the posterior vertex (figure 1). He had no visual disturbances or diplopia. Fundi were normal. Lungs were clear on auscultation with a normal respiratory system examination. Abdominal examination had no organomegaly or free fluid and digital rectal examination was normal.

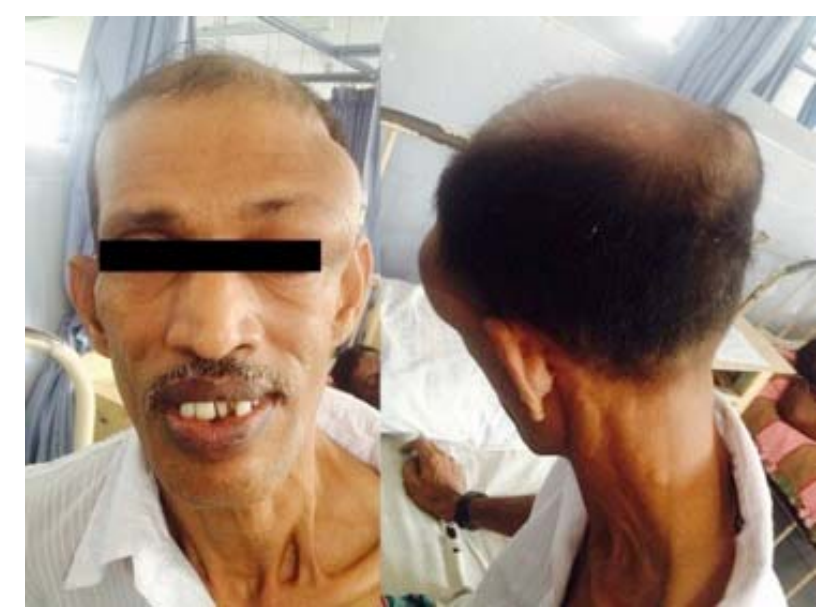

Figure 1. Large bony lumps on frontal and posterior scalp.

On investigation, he had a haemoglobin of $10 \mathrm{~g} / \mathrm{dL}$ with normocytic normochromic red cells, normal white cell count and platelets with no features suggestive of a hematological malignancy, multiple myeloma or lymphoma. His serum ionized calcium level was $2.3 \mathrm{mmol} / \mathrm{l}$ and the serum phosphate level was $1.5 \mathrm{mmol} / \mathrm{l}$ with a marginally high alkaline phosphatase level. ALP was 140 IU/l. S. Parathyroid hormone level was $8.4 \mathrm{pg} / \mathrm{ml}$ which was normal. ESR was 110 in 1st hour and CRP was 10mg/L. Serum protein electrophoresis did not show a monoclonal gammopathy and urine Bens Jones protein was negative. Liver function tests were normal.

Radiograph of right upper leg revealed a clear lytic lesion in the proximal tibia (figure 2) and few more lytic lesions were found on skeletal survey in left humerus (Figure 3). Large bony lumps were clearly seen on Contrast enhanced CT head as irregular lytic bony lesions with intracranial extension invading into brain tissue. Chest radiograph was unremarkable with no focal opacities (figure 4) and ultrasound scan of abdomen was normal. As the diagnosis was still a dilemma, a biopsy from posterior scalp lump was performed and that was reported as inconclusive as the specimen depth was inadequate. Obtaining an adequate biopsy from a scalp lump was difficult due to the intracranial extension and the high vascularity of the lesion.

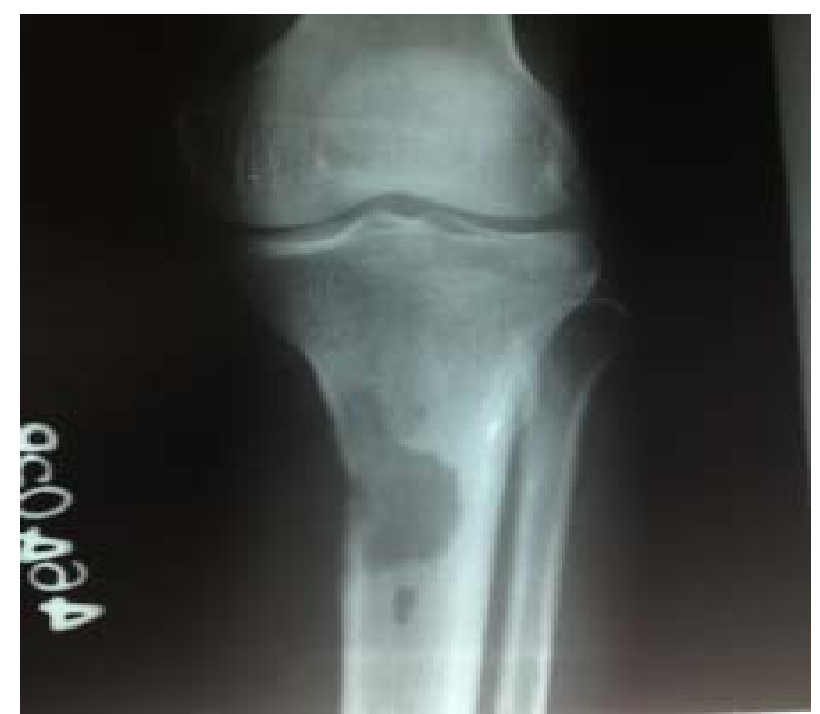

Figure 2. Lytic lesion in the proximal tibia

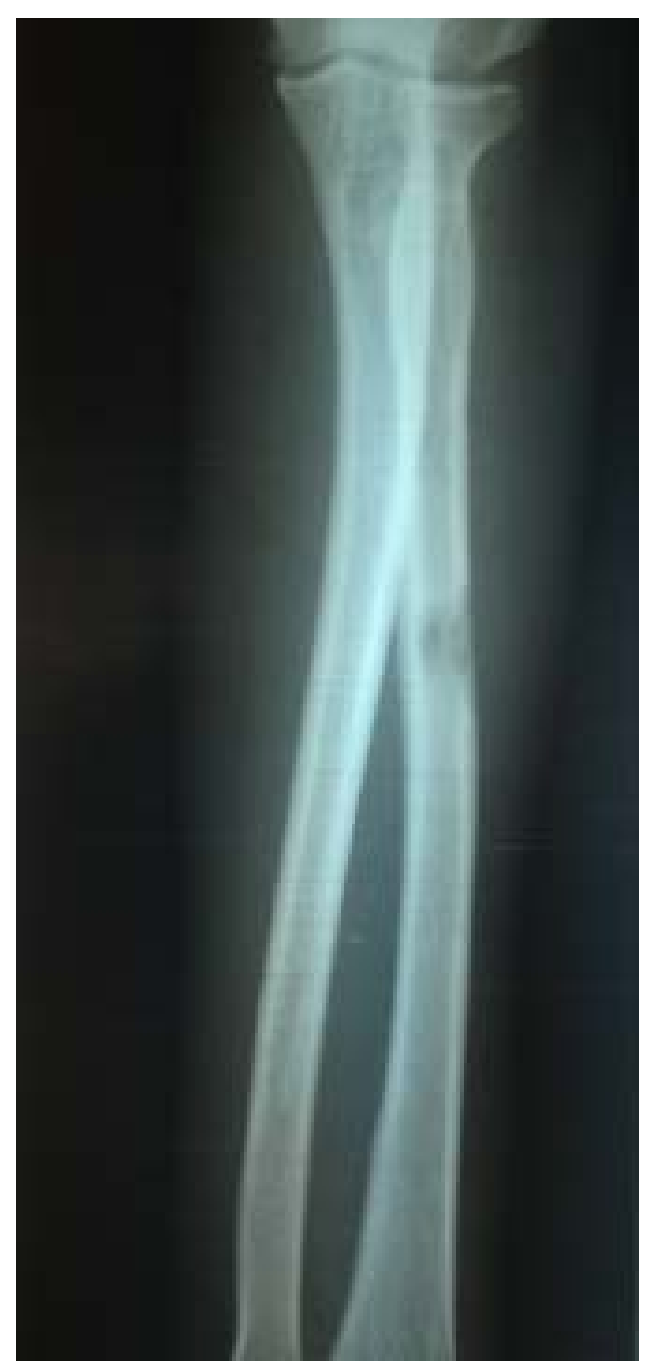

Figure 3. Lytic lesion in left humerus 
Therefore, a contrast enhanced CT (CECT) chest and abdomen was performed in search of a probable primary malignancy which may have given rise to these large metastatic deposits. Surprisingly, this showed a small spiculated left apical lung mass, suggestive of a bronchogenic carcinoma and multiple metastatic deposits on ribs and adrenals (Figure 5). Subsequently, a repeat deep Fine needle aspiration cytology (FNAC) of the posterior scalp lump was performed which revealed possible metastasis from a moderately differentiated squamous cell carcinoma of lung in keeping with the findings on imaging. The patient was directed for further oncological management with chemoradiotherapy.

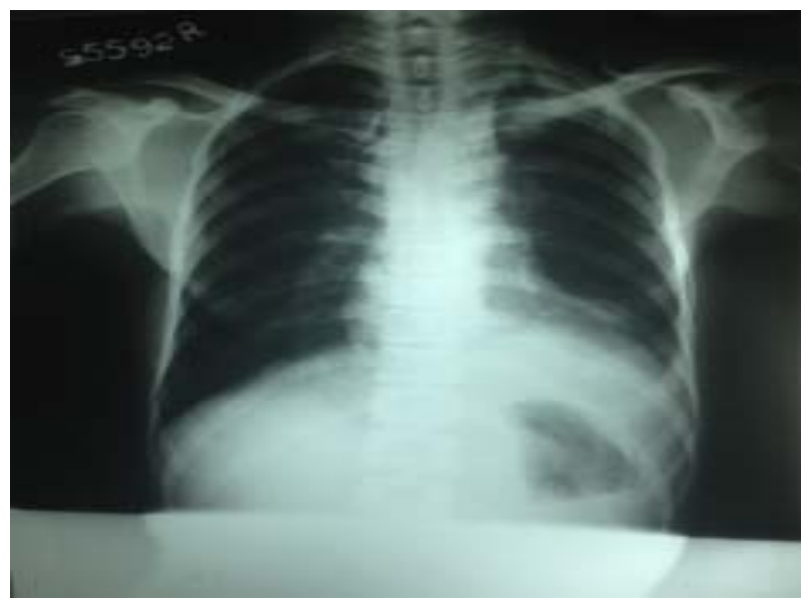

Figure 4. Chest X-ray.

\section{Discussion}

More than $50 \%$ of cancers disseminate to the skeleton, which is the third most frequent site of metastasis. ${ }^{4}$ Bone is also a common site of metastasis from primary lung carcinoma. ${ }^{5}$ Forehead lumps as in our patient, either symptomatic or asymptomatic are a common presentation at the surgical outpatient units than medical units. A significant proportion of subcutaneous lumps of the scalp and forehead are manifestations of malignancies which can be primary or secondary. ${ }^{5}$

With the detection of multiple lytic bone lesions initial differential diagnoses were, a primary bone tumor with multiple deposits, metastasis from internal malignancy or bone cyst forming disease such as Langerhans cell histiocytosis and multiple myeloma. Since the blood picture was not suggestive and the serum protein electrophoresis was normal, multiple myeloma was regarded less likely. In our patient the alarming signs were the rapid progressive enlargement of scalp lumps and the history of loss of appetite and weight. His chronic smoking history too was a possible risk factor for malignancy. Interestingly, there were two case reports found in literature describing a similar presentation with large bony scalp lumps which on further testing, have found to be deposits from a primary hepatocellular carcinoma in one patient ${ }^{6}$, and a plasmacytoma in the other. ${ }^{7}$

The deep FNAC from large posterior scalp lump revealed a metastatic deposit of squamous cell origin. Squamous cell carcinoma of the lung (SqCC) represents $30 \%$ of all non-small cell lung carcinomas (NSCLC). ${ }^{1}$ SqCC arises from dysplasia of the squamous epithelium of the bronchi and is conventionally defined via the histopathologic features of keratinization and intracellular bridges. ${ }^{5}$ Over $50 \%$ of patients with NSCLC have disseminated disease at the time of diagnosis which indicates poor prognosis. ${ }^{3}$

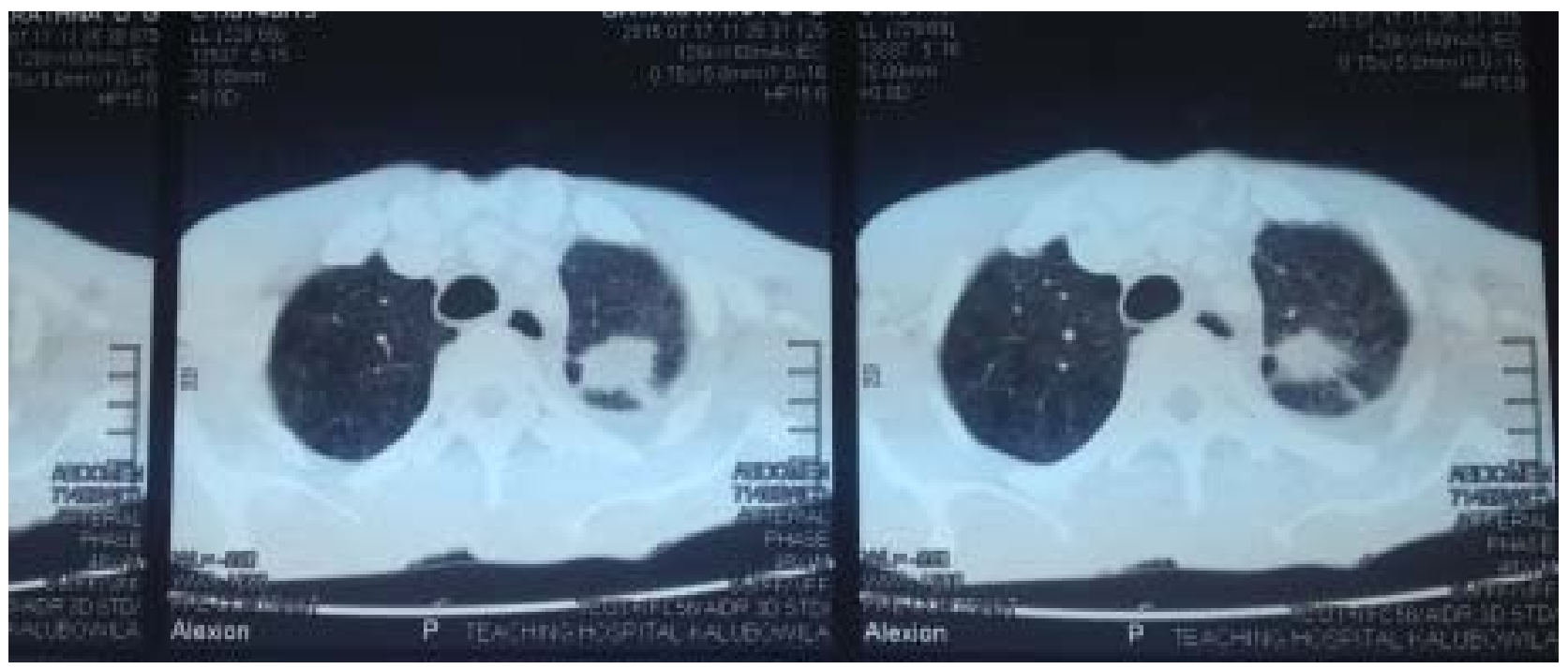

Figure 5. CECT chest showing left side upper lobe spiculated lung mass. 
There are only two case reports on lung carcinoma presenting initially with metastasis to distant bony sites such as mandible ${ }^{3}$, carpal bones ${ }^{1}$, where the diagnosis had been difficult at presentation. But, in both these cases primary lung lesion had been clearly evident on chest radiograph even with the absence of clinical signs. In our patient, the primary lung lesion was not evident on chest radiograph and he had no clinical signs at presentation. There was one case report published in which the primary lung pathology had not been evident on chest $x$-ray like in our patient and was detected on further advanced imaging. ${ }^{5}$ But, there the metastatic deposit had been a solitary painless lump which had not been multiple and extensive as in our patient.

The prognosis of patients with stage IV SqCC is extremely poor with a median survival of four months. ${ }^{2}$ Therefore, treatment of patients in this stage should be focused primarily on palliation. Palliative radiotherapy without surgery has also been used with or without dexamethasone. Also, platinum-based chemotherapy agents, such as cisplatin and carboplatin, are reported to have a $29 \%$ response rate against intra-cranially extending metastatic lesions. ${ }^{2}$ Our patient was directed for oncological management after diagnosis, where the patient refused palliative radiotherapy and was continued on dexamethasone and analgesics.

\section{Conclusion}

We report an extremely rare initial presentation of a primary lung carcinoma with large extensive metastatic bony lumps and bone pain without clear clinical or chest radiographic evidence. This case should serve as an example to have a high degree of suspicion of a lung malignancy in such situations and to have a low threshold to perform early advanced imaging along with possible tissue biopsy, to prevent further diagnostic and therapeutic delay.

\section{Abbreviations}

SqCC - Squamous cell carcinoma, NSCLC - Non small cell lung carcinoma, FNAC - Fine needle aspiration cytology, CECT - Contrast enhanced computed tomography.

\section{Consent}

Written informed consent was obtained from the patient for publication of this case report and any accompanying images. A copy of the written consent is available for review.

\section{Competing interests}

Authors declare that they have no competing interests.

\section{Availability of data and material}

The data and material supporting the conclusions of this article are included within the article and its additional files.

\section{Authors' contribution}

All authors were involved in the management of the patient. DA researched the background literature on the case and wrote the first draft. NS, CL, SS and LF contributed towards the discussions and analysis of the case. All authors read and approved the final manuscript.

\section{Acknowledgements}

No specific acknowledgements.

\section{References}

1. Aboulafia AJ, Aboulafia DM. Incidence, location and diagnostic evaluation of metastatic bone disease. Orthop Clin North Am 2000; 31: 515-28.

2. Imran Kader, Michael Strong and Mathew George. Skull destruction from intracranial metastasis arising from pulmonary squamous cell carcinoma: a case report. Journal of Medical Case Reports 2013; 7: 28.

3. Francesca Maria Giugliano, Domingo Alberti, Giovanna Guida, Giampaolo De Palma, Luciano ladanza, Maria Mormile, Fabrizio Cammarota, Agnese Montanino, Franco Fulciniti, Vincenzo Ravo and Paolo Muto: Non small-cell lung cancer with metastasis to thigh muscle and mandible: two case reports. Journal of Medical Case Reports 2013; 7: 28.

4. Giuseppe Rinonapoli, Auro Caraffa, Renato Antenucci. Lung cancer presenting as a metastasis to the carpal bones: a case report. Journal of Medical Case Reports 2012; 6: 384.

5. García Carretero R, Sanchez-Redondo J, Barrio-Alonso MJ, Lopez-Marti MP. Lung carcinoma presenting as a solitary, painless frontal bone lump. BMJ Case Rep. 2015; 16: 2015.

6. Senapati SB, Mishra SS, Dhir MK, Das S, Tripathy K. A case of multiple myeloma presenting as scalp swelling with intracranial extension. J Neurosci Rural Pract. 2013; 4(4): 445-8.

7. Dong L, Zhang X, Zhang H, Song R, Gu X, He L. Solitary plasmacytoma of the skull: Two case reports. Oncol lett. 2013; 5 (2): 479-82. 